\title{
THE DIRECT PRODUCT AND THE CROSSED PRODUCT OF RINGS OF OPERATORS
}

\author{
TEISHIRÔ SAITÔ
}

(Received March 30, 1959)

Introduction. Recently, the concept of the crossed product of rings of operators has been introduced and studied by T. Turumaru and N. Suzuki [9], [8]. The results in this paper, generally speaking, are concerned with the relationships that exist between the direct products and the crossed products of finite factors, which will be stated in $\$ 2$.

1. Preliminaries. Throughout this paper, we assume that all $W^{*}$-algebras are finite factors with the invariants $C=1$ for the sake of convenience. An isomorphism between $W^{*}$-algebras means a *-isomorphism, and thus an automorphism of a $W^{*}$-algebra is understood as a ${ }^{*}$-automorphism. By a group of outer automorphisms of a $W^{*}$-algebra, we understand as a group of automorphisms all of which are outer automorphisms except the unit.

Next we shall explain the construction of the crossed product of a finite factor by its group of automorphisms (see [8]). Let $\mathbf{M}$ be a finite factor with the invariant $C=1$ on a Hilbert space $\mathbf{H}$ and $G$ a group of automorphisms of $\mathbf{M}$. Let $\phi$ be a separating and generating trace vector for $\mathbf{M}$ and we put $\rho(a)=(a \phi, \phi)$, for all $a \in \mathbf{M}$. For each $\sigma \in G$ we define $u_{\sigma}(a \phi)$ $=a^{\sigma-1} \phi$, for every $a \in \mathbf{M}$, where $a^{\tau}$ is the image of $a$ by an automorphism 7. Then we have $\left\|u_{\sigma}(a \phi)\right\|^{2}=\left\|a^{\sigma-1} \phi\right\|^{2}=\rho\left(\left(a^{*} a\right)^{\sigma^{-1}}\right)=\rho\left(a^{*} a\right)=\|a \phi\|^{2}$, and thus $u_{\sigma}$ can be extended to a unitary operator on $\mathbf{H}$ which is also denoted by $u_{\sigma}$.

Now consider the Hilbert space $\mathbf{H} \otimes l_{2}(G)$. If we choose the complete orthonormal set $\left\{\varepsilon_{\sigma}\right\}_{\sigma, G}$ in $l_{2}(G)$ such as $\varepsilon_{\sigma}(\tau)=1$ for $\tau=\sigma$, and $=0$ otherwise, each vector $\boldsymbol{\phi} \in \mathbf{H} \otimes l_{2}(G)$ is expressed in the form $\boldsymbol{\phi}=\sum_{\sigma_{\epsilon} G} \boldsymbol{\phi}_{\sigma} \otimes \varepsilon_{\sigma}$, where $\boldsymbol{\varphi}_{\sigma} \in \mathbf{H}$ and $\sum_{\sigma \in G}\left\|\boldsymbol{\varphi}_{\sigma}\right\|^{2}<\infty$. We define the operators $\tilde{a}\left(=a^{\sim}\right)$ and $\tilde{u}_{\tau}$ on $\mathbf{H} \otimes l_{2}(G)$ for each $a \in \mathbf{M}$ and $\tau \in G$ by

$$
\begin{aligned}
& \tilde{a}\left(\sum_{\sigma_{\epsilon} G} \boldsymbol{\varphi}_{\sigma} \otimes \varepsilon_{\sigma}\right)=\sum_{\sigma \in G} a \boldsymbol{\varphi}_{\sigma} \otimes \varepsilon_{\sigma}, \text { and } \\
& \tilde{u}_{\tau}\left(\sum_{\sigma \in G} \boldsymbol{\varphi}_{\sigma} \otimes \varepsilon_{\sigma}\right)=\sum_{\sigma \in G} u_{\tau} \boldsymbol{\varphi}_{\sigma} \otimes \varepsilon_{\tau \sigma}
\end{aligned}
$$

for all $\sum_{\sigma \in G} \boldsymbol{\varphi}_{\sigma} \otimes \varepsilon_{\sigma} \in \mathbf{H} \otimes l_{2}(G)$. It is easy to see that $\tilde{u}_{\sigma}^{*} \tilde{a} \tilde{u}_{\sigma}=\tilde{a}^{\sigma}$ for all $a \in \mathbf{M}$ and $\sigma \in G$. The set $\mathfrak{S}$ of all finite linear combinations $\sum_{i} \tilde{a}_{i} \tilde{u}_{\sigma i}\left(a_{i}\right.$ $\left.\in \mathbf{M}, \sigma_{i} \in G\right)$ is a ${ }^{*}$-algebra on $\mathbf{H} \otimes l_{2}(G)$ and the $W^{*}$-algebra generated by 
the system $\subseteq$ is called the crossed product of $\mathbf{M}$ by $G$ and denoted by $(\mathbf{M}, G)$. It is noted that $\phi \otimes \varepsilon_{e} \in \mathbf{H} \otimes l_{2}(G)$ is a separating and generating vector for $(\mathbf{M}, G)$ where $e \in G$ is the unit element.

We shall provide some lemmas.

LEMMA 1. Let $\mathbf{M}$ and $\mathbf{N}$ be finite factors with the invariants $C=1$ and $\theta$ be an isomorphism of $\mathbf{M}$ on $\mathbf{N}$. Let $G_{1}$ and $G_{2}$ be groups of automorphisms of $\mathbf{M}$ and $\mathbf{N}$ respectively and $\eta$ an isomorphism between $G_{1}$ and $G_{2}$ such that

$$
\theta\left(a^{\sigma}\right)=\theta(a)^{\eta(\sigma)} \text { for all } a \in \mathbf{M} \text { and } \sigma \in G_{1} .
$$

Then, the crossed product $\left(\mathbf{M}, G_{1}\right)$ of $\mathbf{M}$ by $G_{1}$ is spatially isomorphic to the crossed product $\left(\mathbf{N}, G_{2}\right)$ of $\mathbf{N}$ by $G_{2}$.

PROOF. If we denote the underlying Hilbert spaces of $\mathbf{M}$ and $\mathbf{N}$ by $\mathbf{H}$ and $\mathbf{K}$ respectively, then, by our hypothesis, there is a linear isometric mapping $u$ from $\mathbf{H}$ to $\mathbf{K}$ such that

$$
\theta(a)=u a u^{-1} \text { for every } a \in \mathbf{M} .
$$

Let $\phi \in \mathbf{H}$ be a separating and generating trace vector for $\mathbf{M}$, then $u \phi=\psi$ $\in \mathbf{K}$ is a separating and generating trace vector for $\mathbf{N}$. Using the traces $\rho_{1}$ and $\rho_{2}$ such as $\rho_{1}(a)=(a \phi, \phi)$ for each $a \in \mathbf{M}$ and $\rho_{2}(a)=(a \psi, \psi)$ for each $a \in \mathbf{N}$, we construct the crossed products $\left(\mathbf{M}, G_{1}\right)$ and $\left(\mathbf{N}, G_{2}\right)$ on the Hilbert spaces $\mathbf{H} \otimes l_{2}\left(G_{1}\right)$ and $\mathbf{K} \otimes l_{2}\left(G_{2}\right)$ respectively. Now we define the correspondence $\tilde{u}$ between $\mathbf{H} \otimes l_{2}\left(G_{1}\right)$ and $\mathbf{K} \otimes l_{2}\left(G_{2}\right)$ as follows: For each $\sum_{\sigma \in G_{1}} \boldsymbol{\phi}_{\sigma} \otimes$ $\varepsilon_{\sigma} \in \mathbf{H} \otimes l_{2}\left(G_{1}\right)$,

$$
\tilde{u}\left(\sum_{\sigma_{G} G_{1}} \boldsymbol{\phi}_{\sigma} \otimes \varepsilon_{\sigma}\right)=\sum_{\eta(\sigma) G_{2}} u \boldsymbol{\varphi}_{\sigma} \otimes \varepsilon_{\eta(\sigma)} .
$$

It is obvious that $\tilde{u}$ is a linear isometric mapping of $\mathbf{H} \otimes l_{2}\left(G_{1}\right)$ on $\mathbf{K} \otimes l_{2}$ $\left(G_{2}\right)$. Next we define the mapping $\tilde{\theta}$ of the crossed product $\left(\mathbf{M}, G_{1}\right)$ to the crossed product $\left(\mathbf{N}, G_{2}\right)$ by

$$
\tilde{\theta}\left(\sum_{i} \tilde{a}_{i} \tilde{u}_{\sigma_{i}}\right)=\sum_{i} \theta\left(a_{j}\right) \tilde{u}_{\eta\left(\sigma_{i}\right)}
$$

for every finite linear combinations $\sum_{i} \tilde{a}_{i} \tilde{u}_{\sigma_{t}} \in\left(\mathbf{M}, G_{1}\right)$. It is easily seen that $u_{\eta(\sigma)}=u u_{\sigma} u^{-1}$ for all $\sigma \in G_{1}$. For any $a, b \in \mathbf{M}$ and $\sigma, \tau \in G_{1}$ we have

$$
\begin{aligned}
\tilde{\theta}\left(\left(\tilde{a} \tilde{u}_{\sigma}\right)^{*}\right) & =\tilde{\theta}\left(\tilde{u}_{\sigma}^{*} \tilde{a}^{*}\right)=\tilde{\theta}\left(\tilde{a}^{* \sigma} \tilde{u}_{\sigma-1}\right) \\
& =\theta\left(a^{* \sigma}\right)^{-} \tilde{u}_{\eta(\sigma-1)}=\theta(a)^{-* \eta(\sigma)} \tilde{u}_{\eta(\sigma)-1}=\tilde{u}_{\eta(\sigma)}^{*} \theta(a)^{-*} \\
& =\left(\tilde{\theta}\left(\tilde{a} \tilde{u}_{\sigma}\right)\right)^{*},
\end{aligned}
$$

and

$$
\tilde{\theta}\left(\left(\tilde{a} \tilde{u}_{\sigma}\right)\left(\tilde{b} \tilde{u}_{\tau}\right)\right)=\tilde{\theta}\left(\tilde{u} \tilde{b}^{\sigma-1} \tilde{u}_{\sigma \tau}\right)=\theta\left(a b^{\sigma-1}\right)^{-} \tilde{u}_{\eta(\sigma \tau)}
$$




$$
\begin{aligned}
& =\theta(a)^{\sim} \theta(b)^{\sim \eta(\sigma)-1} \tilde{u}_{\eta(\sigma) \eta(\tau)}=\theta(a)^{\sim} \tilde{u}_{\eta(\sigma)} \theta(b)^{\sim} \tilde{u}_{\eta(\tau)} \\
& =\tilde{\theta}\left(\tilde{a} \tilde{u}_{\sigma}\right) \tilde{\theta}\left(\tilde{b} \tilde{u}_{\tau}\right) .
\end{aligned}
$$

Moreover we have

$$
\begin{aligned}
\tilde{u}^{-1}\left\{\tilde{\theta}\left(\tilde{a} \tilde{u}_{\sigma}\right)\right\} \tilde{u}\left(\phi \otimes \varepsilon_{e}\right) & =\tilde{u}^{-1}\left(\theta(a) \tilde{u}_{\eta(\sigma)}\right)\left(u \phi \otimes \varepsilon_{\eta(e)}\right) \\
& =\tilde{u}^{-1}\left(\theta(a) u_{\eta(\sigma)} u \phi \otimes \varepsilon_{\eta(\sigma)}\right) \\
& =\tilde{u}^{-1}\left(u a u^{-1} u u_{\sigma} u^{-1} u \phi \otimes \varepsilon_{\eta(\sigma)}\right) \\
& =\tilde{u}^{-1}\left(u a u_{\sigma} \phi \otimes \varepsilon_{\eta(\sigma)}\right) \\
& =\left(\tilde{u} \bar{u}_{\sigma}\right)\left(\phi \otimes \varepsilon_{e}\right),
\end{aligned}
$$

where $e \in G_{1}$ is the unit element. As $\phi \otimes \varepsilon_{e}$ is a separating and generating vector for the crossed product $\left(\mathbf{M}, G_{1}\right)$, we have, for all $\sum_{i} \tilde{a}_{i} \tilde{u}_{\sigma_{i}} \in\left(\mathbf{M}, G_{1}\right)$

$$
\tilde{\theta}\left(\sum_{i} \tilde{a}_{i} \tilde{u}_{\sigma_{i}}\right)=\tilde{u}\left(\sum_{i} \tilde{a}_{i} \tilde{u}_{\sigma_{i}}\right) \tilde{u}^{-1} \text {. }
$$

Hence, by the construction of $\left(\mathbf{M}, G_{1}\right)$ and $\left(\mathbf{N}, G_{2}\right)$, the mapping $\tilde{\theta}$ is extended to a spatial isomorphism between the crossed product $\left(\mathbf{M}, G_{1}\right)$ and the crossed product $\left(\mathbf{N}, G_{2}\right)$.

REMARK. As a special case, we see that if $\theta$ is an isomorphism of $\mathbf{M}$ on $\mathbf{N}$ and if $G_{1}$ is a group of automorphisms of $\mathbf{M}$, then the formula $a^{\sigma_{2}}=$ $\theta\left[\left\{\theta^{-1}(a)\right\}^{\sigma_{1}}\right]$ for each $a \in \mathbf{N}$, defines an automorphism of $\mathbf{N}$ for each $\sigma_{1} \in G_{1}$ and $G_{2}=\left\{\sigma_{2}\right\}$ satisfies the condition in Lemma 1 .

The following lemma is derived from [1: Chap. 1, \$4, Prop. 2], and we omit the proof.

LEMMA 2. If $\mathbf{M}, \mathbf{N}$ are finite factors with the invariants $C=1$, and $G_{1}, G_{2}$ are groups of automorphisms of $\mathbf{M}, \mathbf{N}$ respectively, then the direct product $G=G_{1} \times G_{2}$ admits a faithful representation as a group of automorphisms of the direct product of $\mathbf{M}$ and $\mathbf{N}$, in which for any $a \in \mathbf{M}, b \in$ $\mathbf{N}$, and $\sigma=\left(\sigma_{1}, \sigma_{2}\right) \in G,(a \otimes b)^{\sigma}=a^{\sigma_{1}} \otimes b^{\sigma_{2}}$.

2. The theorems. In this section we shall prove the results stated in the introduction. The following theorem shows the commutativity of the crossed product operation and the direct product operation.

THEOREM 1. Let $\mathbf{M}, \mathbf{N}$ be finite factors with the invariants $C=1$ and $G_{1}, G_{2}$ groups of automorphisms of $\mathbf{M}, \mathbf{N}$ respectively, then $\left(\mathbf{M} \otimes \mathbf{N}, G_{1} \times G_{2}\right)$ is isomorphic to $\left(\mathbf{M}, G_{1}\right) \otimes\left(\mathbf{N}, G_{2}\right)$, where $G_{1} \times G_{2}$ is the group of automorphisms of $\mathbf{M} \otimes \mathbf{N}$ assured in Lemma 2.

ProOF. Suppose that $\mathbf{M}$ and $\mathbf{N}$ act on Hilbert spaces $\mathbf{H}$ and $\mathbf{K}$ respectively. Let $\phi_{1}\left(\right.$ resp. $\left.\phi_{2}\right)$ be a separating and generating trace vector for $\mathbf{M}$ (resp. 
$\mathbf{N})$. Then $\phi=\phi_{1} \& \phi_{2}$ is a separating and generating trace vector for $\mathbf{M} \otimes$ N. Considering the traces $\rho_{1}(a)=\left(a \phi_{1} \phi_{1}\right)$ for $a \in \mathbf{M} . \rho_{2}(b)=\left(b \phi_{2}, \phi_{2}\right)$ for $b$ $\in \mathbf{N}$, and $\rho(x)=(x \phi, \phi)$ for $x \in \mathbf{M} \otimes \mathbf{N}$, we construct the crossed products $\left(\mathbf{M}, G_{1}\right),\left(\mathbf{N}, G_{2}\right)$ and $\left(\mathbf{M} \otimes \mathbf{N}, G_{1} \times G_{2}\right)$. The underlying Hilbert spaces of these crossed products are $\mathbf{H} \otimes l_{2}\left(G_{1}\right), \mathbf{K} \otimes l_{2}\left(G_{2}\right)$ and $(\mathbf{H} \otimes \mathbf{K}) \otimes l_{2}\left(G_{1} \times G_{2}\right)$ respectively. It is known the existence of linear isometric mapping $\tilde{v}$ from $\left(\mathbf{H} \otimes l_{2}\left(G_{1}\right)\right) \otimes\left(\mathbf{K} \otimes l_{2}\left(G_{2}\right)\right)$ onto $(\mathbf{H} \otimes \mathbf{K}) \otimes\left(l_{2}\left(G_{1}\right) \otimes l_{2}\left(G_{2}\right)\right)$ such that

$$
\tilde{v}((\phi \otimes f) \otimes(\psi \otimes g))=(\boldsymbol{\varphi} \otimes \psi) \otimes(f \otimes g)
$$

for $\boldsymbol{\phi} \in \mathbf{H}, \psi \in \mathbf{K}, f \in l_{2}\left(G_{1}\right)$ and $g \in l_{2}\left(G_{2}\right)$. Choosing a complete orthonormal sets $\left\{\varepsilon_{\sigma}\right\}_{\sigma \in G_{1} \times G_{2}}\left\{\varepsilon_{\sigma_{1}}\right\}_{\sigma_{1 \epsilon} G_{1}}$ and $\left\{\varepsilon_{\sigma_{2}}\right\}_{\sigma_{2} \in G_{2}}$ of $l_{2}\left(G_{1} \times G_{2}\right), l_{2}\left(G_{1}\right)$ and $l_{2}\left(G_{2}\right)$ respectively, we define a mapping $u_{0}$ of $l_{2}\left(G_{1} \times G_{2}\right)$ to $l_{2}\left(G_{1}\right) \otimes l_{2}\left(G_{2}\right)$ by

$$
u_{0}\left(\varepsilon_{\sigma}\right)=\varepsilon_{\sigma_{1}} \otimes \varepsilon_{\sigma_{2}} \quad \text { for each } \sigma=\left(\sigma_{1}, \sigma_{2}\right) \in G_{1} \times G_{2} .
$$

As $\left\{\varepsilon_{\sigma_{1}} \otimes \varepsilon_{\sigma_{2}}\right\}_{\sigma_{1}-G_{1}, \sigma_{2} G_{2}}$ constitutes a complete orthonormal set of $l_{2}\left(G_{1}\right) \otimes$ $l_{2}\left(G_{2}\right), u_{0}$ can be extended to a linear isometric mapping $u$ of $l_{2}\left(G_{1} \times G_{2}\right)$ on $l_{2}\left(G_{1}\right) \otimes l_{2}\left(G_{2}\right)$. Thus using these mappings $\tilde{v}$ and $u$, we define a correspondence $\tilde{u}_{0}$ of $(\mathbf{H} \otimes \mathbf{K}) \otimes l_{2}\left(G_{1} \times G_{2}\right)$ and $\left(\mathbf{H} \otimes l_{2}\left(G_{1}\right)\right) \otimes\left(\mathbf{K} \otimes l_{2}\left(G_{2}\right)\right)$ as follows. For each $\boldsymbol{\phi} \in \mathbf{H} \otimes \mathbf{K}$ and $f \in l_{2}\left(G_{1} \times G_{2}\right)$,

$$
\tilde{u}_{0}(\varphi \otimes f)=\tilde{v}^{-1}(\varphi \otimes u f) \text {. }
$$

Being isometric, $\tilde{u}_{0}$ can be extended to a linear isometric mapping $\tilde{u}$ of $(\mathbf{H} \otimes \mathbf{K}) \otimes l_{2}\left(G_{1} \times G_{2}\right)$ on $\left(\mathbf{H} \otimes l_{2}\left(G_{1}\right)\right) \otimes\left(\mathbf{K} \otimes l_{2}\left(G_{2}\right)\right)$. Finally we shall show that $\tilde{u}$ induces an isomorphism between $\left(\mathbf{M} \otimes \mathbf{N}, G_{1} \times G_{2}\right)$ and $\left(\mathbf{M}, G_{1}\right) \otimes$ $\left(\mathbf{N}, G_{2}\right)$. To this end we define the mapping $\tilde{\theta_{0}}$ from $\left(\mathbf{M}, G_{1}\right) \otimes\left(\mathbf{N}, G_{2}\right)$ to $\left(\mathbf{M} \otimes \mathbf{N}, G_{1} \times G_{2}\right)$ as follow : For every $\sum_{i} \tilde{a}_{i} \tilde{u}_{\sigma_{1 i}} \in\left(\mathbf{M}, G_{1}\right)$ and $\sum_{j} \tilde{b}_{j} \tilde{u}_{\sigma_{2},}$ $\in\left(\mathbf{N}, G_{2}\right)$,

$$
\begin{gathered}
\tilde{\theta}_{0}\left(\left(\sum_{i} \tilde{a}_{i} \tilde{u}_{\sigma_{1 i}}\right) \otimes\left(\sum_{j} \tilde{b}_{j} \tilde{u}_{\sigma_{2}}\right)\right)=\tilde{\theta}_{0}\left(\sum_{i, j} \tilde{a}_{i} \tilde{u}_{\sigma_{1 i}} \otimes \tilde{b}_{j} \tilde{u}_{\sigma_{2},}\right) \\
=\sum_{j, i}\left(a_{i} \otimes b_{j}\right) \tilde{u}_{\left(\sigma_{1,}, \sigma_{2 j}\right)} \in\left(\mathbf{M} \otimes \mathbf{N}, G_{1} \times G_{2}\right) .
\end{gathered}
$$

Then for any $\tilde{a} \tilde{u}_{\sigma_{1}}, \tilde{c} \tilde{u}_{\tau_{1}} \in\left(\mathbf{M}, G_{1}\right)$ and $\tilde{b} \tilde{u}_{\sigma_{2}}, \tilde{d} \tilde{u}_{\tau_{2}} \in\left(\mathbf{N}, G_{2}\right)$, we have

$$
\begin{gathered}
\tilde{\theta}_{0}\left(\left(\tilde{a} \tilde{u}_{\sigma_{1}} \otimes \tilde{b} \tilde{u}_{\sigma_{2}}\right)^{*}\right)=\tilde{\theta}_{0}\left(\tilde{u}_{1_{1}}^{*} \tilde{u}^{*} \otimes \tilde{u}_{\sigma_{2}}^{*} \tilde{b}^{*}\right)=\tilde{\theta}_{0}\left(\tilde{a}^{* \sigma_{1}} \tilde{u}_{\sigma_{1}-1} \otimes \tilde{b}^{* \sigma_{2}} \tilde{u}_{\sigma_{2}-1}\right) \\
=\left(a^{\sigma_{1}} \otimes b^{\sigma_{2}}\right)^{-*} \tilde{u}_{\left(\sigma_{1}, \sigma_{2}\right)^{-1}}=(a \otimes b)^{-\sigma *} \tilde{u}_{\left(\sigma_{1}, \sigma_{2}\right)}^{*}=\tilde{u}_{\left(\sigma_{1}, \sigma_{2}\right)}^{*}(a \otimes b)^{-*} \\
=\left((a \otimes b)^{-} \tilde{u}_{\left(\sigma_{1}, \sigma_{2}\right)}\right)^{*}=\left(\tilde{\theta}_{0}\left(\tilde{u} \tilde{u}_{\sigma_{1}} \otimes \tilde{b} \tilde{u}_{\sigma_{2}}\right)\right)^{*},
\end{gathered}
$$

and

$$
\begin{aligned}
& \tilde{\theta}_{0}\left(\left(\tilde{a} \tilde{u}_{\sigma_{1}} \otimes \tilde{b} \tilde{u}_{\sigma_{2}}\right)\left(\tilde{c} \tilde{u}_{\tau_{1}} \otimes \tilde{d} \tilde{u}_{\tau_{2}}\right)\right)=\tilde{\theta}_{0}\left(\tilde{a} \tilde{c}^{\sigma_{1}-1} \tilde{u}_{\sigma_{1} \tau_{1}} \otimes \tilde{b} \tilde{d}^{\sigma_{2}-1} \tilde{u}_{\sigma_{2} \tau_{2}}\right) \\
& \quad=(a \otimes b)^{-}(c \otimes d)^{-\left(\sigma_{1}, \sigma_{2}\right)^{-1}} \tilde{u}_{\left(\sigma_{1}, \tau_{1}\right)\left(\sigma_{2}, \tau_{2}\right)}=(a \otimes b)^{-} \tilde{u}_{\left(\sigma_{1}, \sigma_{2}\right)}(c \otimes d)^{-} \tilde{u}_{\left(\tau_{1}, \tau_{2}\right)} \\
& \quad=\tilde{\theta}_{0}\left(\tilde{a} \tilde{u}_{\sigma_{1}} \otimes \tilde{b} \tilde{u}_{\sigma_{2}}\right) \tilde{\theta}_{0}\left(\tilde{c} \tilde{u}_{\tau_{1}} \otimes \tilde{d} \tilde{u}_{\tau_{2}}\right) .
\end{aligned}
$$


Moreover, noting $u_{\left(\sigma_{1}, \sigma_{2}\right)}\left(\phi_{1} \otimes \phi_{2}\right)=u_{\sigma_{1}} \phi_{1} \otimes u_{\sigma_{2}} \phi_{2}$, we get

$$
\begin{aligned}
\tilde{u}^{-1}\left(\tilde{a} \tilde{u}_{\sigma_{1}}\right. & \left.\otimes \tilde{b} \tilde{u}_{\sigma_{2}}\right) \tilde{u}\left(\phi \otimes \varepsilon_{\left(e_{1}, e_{2}\right)}\right) \\
& \left.=\tilde{u}^{-1}\left(\tilde{a} \tilde{u}_{\sigma_{1}} \otimes \tilde{b} \tilde{u}_{\sigma_{2}}\right)\left(\phi_{1} \otimes \varepsilon_{e_{1}}\right) \otimes\left(\phi_{2} \otimes \varepsilon_{e_{2}}\right)\right) \\
& =\tilde{u}^{-1}\left(\left(a u_{\sigma_{1}} \phi_{1} \otimes \varepsilon_{\sigma_{1}}\right) \otimes\left(b u_{\sigma_{2}} \phi_{2} \otimes \varepsilon_{\sigma_{2}}\right)\right) \\
& =\left(a u_{\sigma_{1}} \phi_{1} \otimes b u_{\sigma_{2}} \phi_{2}\right) \otimes \varepsilon_{\left(\sigma_{1}, \sigma_{2}\right)} \\
& \left.=(a \otimes b)^{-} \tilde{u}_{\left(\sigma_{1}, \sigma_{2}\right)}\right)\left(\phi \otimes \varepsilon_{\left(e_{1} e_{2}\right)}\right)=\tilde{\theta}_{\theta}\left(\tilde{u} \tilde{u}_{\sigma_{1}} \otimes \tilde{b} \tilde{u}_{\sigma_{2}}\right)\left(\phi \otimes \varepsilon_{\left(e_{1}, e_{2}\right)}\right),
\end{aligned}
$$

where $e_{1} \in G_{1}$ (resp. $e_{2} \in G_{2}$ ) is the identity of $G_{1}$ (resp. $G_{2}$ ). Thus, as $\phi \otimes$ $\varepsilon_{\left(e_{1}, e_{2}\right)}$ is a seperating and generating vector for $\left(\mathbf{M} \otimes \mathbf{N}, G_{1} \times G_{2}\right)$, we obtain

$$
\tilde{u}^{-1}\left(\tilde{a} \bar{u}_{\sigma_{1}} \otimes \tilde{b} \bar{u}_{\sigma_{2}}\right) \tilde{u}=\tilde{\theta}_{0}\left(\tilde{a} \bar{u}_{\sigma_{1}} \otimes \tilde{b} \tilde{u}_{\sigma_{2}}\right) \text {. }
$$

Hence $\tilde{\theta}_{0}$ can be extended to an isomorphism $\tilde{\theta}$ of $\left(\mathbf{M}, G_{1}\right) \otimes\left(\mathbf{N}, G_{2}\right)$ and $\left(\mathbf{M} \otimes \mathbf{N}, G_{1} \times G_{2}\right)$, and the proof is completed.

Let $\mathbf{M}$ be an approximately finite factor on a separable Hilbert space with the invariant $C=1$ and $G$ a group of automorphisms of $\mathbf{M}$. It is known that $\mathbf{M}$ is the direct product of two approximately finite factors $\mathbf{M}_{1}$ and $\mathbf{M}_{2}$ on separable Hilbert spaces with the invariants $C=1$ (cf. [4: Lemma 5.2.1] and [6: Lemma 4]). As remarked after Lemma 1, $G$ admits a faithful representation $G^{\prime}$ as a group of automorphisms such as the crossed product $(\mathbf{M}, G)$ is isomorphic to the crossed product $\left(\mathbf{M}_{2}, G^{\prime}\right)$. Now we consider the identity automorphism $e$ of $\mathbf{M}_{1}$ in which every element of $\mathbf{M}_{1}$ remains in place and observe that $\mathbf{M}_{1}$ is isomorphic to the crossed product $\left(\mathbf{M}_{1}, e\right)$. Then, by Theorem 1 , the direct product $\mathbf{M}_{1} \otimes\left(\mathbf{M}_{2}, G^{\prime}\right)$ is isomorphic to the crossed product $\left(\mathbf{M}, e \times G^{\prime}\right)$. It is obvious that $e \times G^{\prime}$ is isomorphic to $G$.

On the other hand, as shown in [8], the crossed product $(\mathbf{M}, G)$ is a factor of type $\mathbf{I I}_{1}$ if $G$ is a group of outer automorphisms of $\mathbf{M}$, and then $\left(\mathbf{M}_{2}, G^{\prime}\right)$ is also a factor of type $\mathbf{I I}_{1}$. Hence, as $\mathbf{M}_{1}$ has the property $\boldsymbol{\Gamma}, \mathbf{M}_{1}$ $\otimes\left(\mathbf{M}_{2}, G^{\prime}\right)$ is a factor of type $\mathbf{I I}_{1}$ with the property $\Gamma$ by [2: Theorem 2] if $G$ is a group of outer automorphisms of $\mathbf{M}$.

Furthermore it is proved in [7] that an arbitrary countable group is isomorphic to a group of outer automorphisms of the approximately finite factor on a separable Hilbert space.

Summing up the above we obtain

THEOREM 2. Let $\mathbf{M}$ be the approximately finite factor on a separable Hilbert space and $G_{0}$ an arbitrary countable group, then there exists a group $G$ of automorphisms of $\mathbf{M}$, isomorphic to $G_{0}$, such that the crossed product $(\mathbf{M}, G)$ is a factor of type $\mathbf{I I}_{1}$ with the property $\mathbf{\Gamma}$. 


\section{REFERENCES}

[1] J, DIXMIER, Les algèbres d'opérateurs dans l'espace hilbertien, Paris, (1957).

[2] Y. MisonoU, On divisors of factors, Tôhoku Math. Journ., 8(1956), 63-69.

[3] F J. MURRAY AND J. VON NEUMANN, On rings of operators, Ann. of Math., 37(1936), 116-229.

[4] F. J. MURRAY AND J. VON NEUMANN, On rings of operators IV, Ann. of Math., 44 (1943), 716-808.

[5] J. VON NEUMANN, On infinite direct products, Compositio Mathematica, 6(1938), 1-77.

[6] T.SAITô, On incomplete infinite direct product of $W^{*}$-algebras, Tôhoku Math. Journ., 10(1958), 165-171.

[7] N. SUZUKI, A linear representation of a countably infinite group, Proc. Japan Acad., 34(1958), 575-579.

[8] N.SUZUKI, Crossed products of rings of operators, Tôhoku Math. Journ., 11(1959), 113-124.

[9] T. TURUMARU, Crossed product of operator algebra, Tôhoku Math. Journ., 10(1958), 355-364.

MATHFMATICAL. INSTITUTE, TÔHOKU UNIVERSiTY. 